\title{
Perfiles de Comunicación en Google Scholar Metrics, índice h y nuevas estrategias de difusión de la investigación
}

\author{
Miguel TúÑEZ LóPEZ \\ Universidad de Santiago de Compostela \\ miguel.tunez@usc.es
}

\begin{abstract}
Resumen:
Google Scholar Metrics permite, desde 2012, que los investigadores creen un perfil con su producción científica y académica enlazada en la red y evaluada con indicadores bibliométricos $i h, i 10$ e $i h 5$ personalizados, que referencian la correlación que hay entre la productividad de un investigador y el éxito que aprecian sus pares. Este artículo refleja los resultados de una investigación sobre el uso del perfil, medias de cita, indicadores h referenciales y soportes de citación habituales de investigadores del área de Comunicación en España. También se aproxima a las nuevas estrategias de difusión de la investigación que se derivan del uso del índice $h$ como indicador frente al tradicional factor de impacto.
\end{abstract}

Palabras clave: índice h; factor de impacto; Google Scholar Metrics; revistas científicas; ae-ic; GSM.

\section{Communication profiles in Google Scholar Metrics, h index and new research dissemination strategies}

\begin{abstract}
:
Since 2012, Google Scholar Metrics allows researchers to create an online citations profile where their scientific and academic publications are assessed with personalised bibliometric indicators such as the ih index, the $i 10$ index and the $i h 5$ index, which refer to the relation between a researchers' publications and their importance according to peers. This article presents the results of a research study about the use of the Google Scholar profile, the citation metrics, the $\mathrm{h}$ indicators, and the traditional citations counting tools among Communication researchers in Spain. This study also explores the new research dissemination strategies that have derived from the use of the $\mathrm{h}$ index in comparison to the impact factor.
\end{abstract}

Key Words: h-index; impact factor; Google Scholar Metrics; scientific journals; ae-ic; GSM.

\section{Referencia normalizada:}

Túñez López, M. (2014): Perfiles de comunicación en Google Scholar Metrics, índice h y nuevas estrategias de difusión de la investigación. Historia y Comunicación Social. Vol. 19. Núm. Especial Marzo. Págs. 15-26.

Sumario: 1. Gestión de la comunicación de la investigación difundida. 2. Los indicadores personales en abierto: perfil en GSM 3. Índice h de Comunicación. 4. Metodología. 5. Resultados. 5.1. Niveles de edición. 5.2. Trayectorias como investigador y media de citas. 5.3. Índices h y h5. 5.4. Producción. 5.5. El formato: libro\&artículo. 6. Conclusiones. 7. Bibliografía. 


\section{Gestión de la comunicación de la investigación difundida}

La medición de la difusión investigadora se ha canalizado tradicionalmente a través del factor de impacto $(f i)$ de las revistas aunque en los últimos años el índice $\mathrm{h}$ (ih) ha ido ganado terreno como indicador bibliométrico y ha sido incorporado a sus métricas por Thomson Reuters y por Elsevier. Propuesto en 2005 por J. Hirsch, representa el número $h$ de publicaciones de un autor que han recibido un número mínimo $h$ de citas. El $f i$ se remonta a 1963, cuando Eugene Garfield e Irving Sher proponen evaluar la influencia de las revistas científicas a través del recuento del número de citas que han obtenido en otras revistas de calidad en un período concreto de tiempo, inicialmente los dos años anteriores al de medición.

El área de Comunicación en España no comienza a interesarse por el ih hasta que en 2012 Google Scholar Metrics irrumpe en competencia directa con Web of Knowledge (WoK, Thomson Reuters) y con Scopus (Elsevier) para ofrecer sus propias mediciones de revistas y la posibilidad de que los investigadores creen sus perfiles con indicadores $h$ individuales, a modo de bibliometría personal. En foros científicos del área de Comunicación (exceptuando reuniones de editores de revistas) la primera referencia sobre Google Scholar Metrics se detecta en el IV Congreso de la Sociedad Latina de Comunicación, en diciembre de 2012, con la aportación de Sánchez "Actualización del Î́ndice H de las revistas de Comunicación, según Google Scholar Metrics". Las primeras discusiones sobre el índice h en encuentros científicos de Comunicación aún se hacen esperar hasta mayo de 2013, durante el segundo Congreso de la sección de Métodos de Investigación de la Asociación Española de Investigación en Comunicación (AE-IC)', promovido por la Universidad de Valladolid a principios de mayo de 2013 (Túñez y De Pablos, 2013).

La aceptación del íh como referente de calidad de la difusión de investigación se centra en que combina productividad con impacto. También supone una reinterpretación del valor de los artículos en función del soporte de publicación: el $f i$ es colectivo porque es un indicador de la revista y se proyecta sobre los artículos que ésta incluye; el ih es individual o colectivo ya que mide la aceptación de cada trabajo publicado a través de las citas que recibe y se colectiviza al incluir el artículo para ser evaluado en un conjunto de publicaciones de su/s autor/es o de una revista.

Si el $f i$ depende de las citas que reciban los artículos de una publicación es lógico pensar que las revistas promuevan acciones de visibilidad de sus contenidos entre la comunidad científica de su área; del mismo modo, si el ih es un indicador que aplica a personas y a revistas (o grupos de investigación) es deducible que las acciones de visibilidad de la investigación difundida también pasen a ser una corresponsabilidad que encaja con lo que ha comenzado a referirse como un incipiente marketing de investigación 2.0 (Túñez, 2012) porque da respuesta a la necesidad de planificar nuevas estrategias de visibilidad que permitan abordar la difusión de resultados de investigación como una tarea de gestión integral de la Comunicación. 
Lo que varía es la actitud del investigador. La difusión no finaliza cuando el artículo es aceptado para ser publicado; a partir de ahí se abriría una nueva fase compartida por autor y revista como usuarios proactivos con interactividad online y acciones en red. En esta tarea de visibilizar la investigación difundida se identifican como acciones y/o herramientas tanto el envío del texto por correo electrónico a otros investigadores como la cesión del archivo en $p d f$ (si se ha publicado en un sistema de acceso abierto) al repositorio de la Universidad. También es recomendable programar acciones de difusión con enlace al texto en las webs personales o de grupos de investigación y en las webs para encuentro de investigadores o para intercambio de artículos (desde $p d f$ hasta vídeos) que operan como redes sociales de ámbitos profesionales o científicos como Academica.edu, Researchgate.net, Linkedin, Scivee.tv, Sciencestage.com, biomedeexperts.com, Doc2doc.com, Mendeley.com, Connotea.org, Citulike.org o, incluso, slideshare.net por ejemplo. También se ha derivado la acción de grupos o de instituciones hacia las redes generalistas como un complemento de visibilidad o de proyección social de los resultados de investigación y de otras acciones o servicios del grupo. El propio CSIC, por ejemplo, maneja su perfil en Facebook.

Gestionar la comunicación de investigación publicada también supone, por ejemplo, considerar la utilidad de usar el enlace a los perfiles personales como tarjeta de presentación de la actividad investigadora incorporando la url del perfil a la firma del correo electrónico, en otros perfiles, en los currículos online, en las presentaciones de comunicaciones o posters a congresos y en las charlas o conferencias en ámbitos científicos.

Google abrió camino al crear Google Scholar en 2004 y dio un paso más al ofrecer en 2012 Google Scholar Metrics (GSM) con indicadores bibliométricos de revistas científicas y con puertas abiertas para que los investigadores creasen un perfil personal con su producción científica y académica enlazada en la red y evaluada con indicadores bibliométricos $h$ personalizados. La oferta de Google se decantaba por el ih como referente de impacto de las publicaciones científicas en un contexto en el que la referencia universal era el $f$. El desafío era triple: entrar en el terreno de los indicadores bibliométricos reservado casi en exclusiva a Thomson Reuters (Web of Knowledge) y Elsevier (Scoups); decantarse por el índice de Hirsch como cuantificador de producción e impacto; y ofrecer sus listados gratuitamente y en abierto para todos los internautas.

\section{Los indicadores personales en abierto: perfil en GSM}

GSM empieza en abril de 2012 a ofertar datos de ih con un primer ranking de revistas por lenguas. En noviembre corrige algunos errores y ofrece, además, un listado por áreas temáticas de revistas en inglés. Los datos se renovaron en julio de 2013. Google permite, además, crear perfiles personales o de grupos. El perfil es una herramienta para ordenar y agrupar las publicaciones, mejorar la visibilidad y el 
impacto, gestionar y mantener actualizado el currículo, tener indicadores bibliométricos personales, saber quien cita a quien, recibir alertas y seguir a otros investigadores. Se pueden editar los datos del artículo y del autor pero las citas no son editables ni modificables. Tampoco se puede referenciar el impacto de las citas sin autocitas.

En su corto recorrido, GSM ha sido acogido de manera controvertida porque no explica los criterios con los que ha creado su listado de áreas y disciplinas para agrupar las revistas; porque no revela en cuantas áreas se ha incluido a una revista; y porque no tiene en cuenta el soporte en el que se produce la cita y valida todas las referencias que detectan sus motores de rastreo. GSM es "un producto inmaduro, que consta de múltiples limitaciones en su configuración actual para la evaluación de las revistas científicas" pero también es "una mina de información científica prodigiosa [...] especialmente de utilidad para los científicos de Humanidades y Ciencias Sociales porque controla como nadie lo ha hecho hasta ahora la literatura no anglosajona y aquella transmitida por medios distintos a las revistas científicas, que es la peor controlada por los sistemas de información dominantes en el mundo académico" (Delgado et al, 2012: 4).

Google apuesta por el ih, cuyo punto más fuerte es que se basa en que une la productividad de un investigador y el éxito que aprecian sus pares. Entre las consideraciones negativas destacan que no discrimina en niveles intermedios ya que no tiene en cuenta las citas de reciben los artículos con un número de referencias superiores a $h$; que no sirve para diferenciar entre una carrera emergente y una carrera en declive y que promociona a productores masivos de artículos frente a productores selectivos de pocos textos pero muy referenciados.

Para contribuir a corregir estas distorsiones se han identificado indicadores de entorno. Los incluidos en los perfiles de GSM son: el índice $h 5$, que refiere las citas recibidas por los artículos de un autor en los últimos cinco años naturales completos y el índice i10, o número de artículos de un autor que han recibido, cada uno, al menos diez citas.

\section{3. Índice $\mathbf{H}$ de comunicación}

Elsevier, Thomson Reuters y GSM usan universos de referencia diferentes para obtener sus indicadores por lo que el $i h$ de un mismo investigador o un mismo equipo puede representarse con dígitos diferentes según el patrón de búsqueda que se utilice. La comparativa entre Google, Thomson Reuters y Elsevier que Delgado y Repiso realizan sobre la base del listado de Google de noviembre de 2012 deja clara esa diferencia: Identifican 664 revistas de comunicación en todo el mundo de las que casi la mitad aparecen la lista de GSM de noviembre 2012, pero en Scopus solo están una de cada cuatro y en $W o K$ no llegan a 2 de cada diez (2013:48). La supremacía anglosajona en WoK y Scopus queda visiblemente amortiguada en Google y la difusión en español casi se triplica en GSM sobre las otras dos bases. GSM es la que indexa más 
revistas, de más países y en más idiomas y "mide las revistas de forma muy parecida a los clásicos sistemas de evaluación de revistas (WoK y Scopus), por lo que en líneas generales, y a efectos únicamente de ranking, es una alternativa tan fiable y válida como éstos para medir el impacto de las revistas" (Delgado y Repiso, 2013:51).

\begin{tabular}{|c|c|c|c|c|}
\hline \multicolumn{2}{|c|}{ COMUNICACIÓN (664 revistas) } & GSM (Google) & $\begin{array}{c}\text { Scopus } \\
\text { (Elsevier) }\end{array}$ & $\begin{array}{c}\text { WoS } \\
\text { (T.Reuters) }\end{array}$ \\
\hline \multirow{6}{*}{ Revistas } & $277(41,71 \%)$ & 167 & 106 \\
$(25,15 \%)$ & $(15,96 \%)$ \\
\hline \multirow{6}{*}{ Idiomas } & Países & 30 & 16 & 23 \\
\cline { 2 - 5 } & Todos & 13 & 7 & 7 \\
\cline { 2 - 5 } & Revistas en Inglés & $59,67 \%$ & $88,66 \%$ & $86,92 \%$ \\
\cline { 2 - 5 } & Revistas en Español & $13,77 \%$ & $5,15 \%$ & $5,61 \%$ \\
\hline
\end{tabular}

Tabla 01. Revistas de Comunicación. Fuente: Delgado y Repiso, 2013. Elaboración propia.

El ih no es un indicador válido para establecer comparativas entre diferentes áreas de conocimiento pero sí se considera viable determinar un $h_{R}$ de referencia (Imperial y Rodríguez-Navarro, 2007). Tomando como base el $f i$ de todas las revistas incluidas en In-recs en 2011, el índice h referencial en el área de Comunicación en España sería 17 (Túñez, 2013). El dígito de la revista española de Comunicación con mejor índice h en Scopus es 3; en GSM, y en las aportaciones del grupo EC3 es 13 (Repiso y Delgado, 2013).

Imperial y Rodríguez-Navarro (2007) advertían que $h$, no es aplicable, salvo alguna excepción, a las Ciencias Sociales y a las Humanidades, porque estas áreas carecen de bases de datos de revistas suficientemente extensas y por el gran peso que en estas áreas tiene la publicación de libros. Sin embargo, desde entonces han pasado 6 años caracterizados por un aumento del número de publicaciones científicas y por una creciente tendencia de las referencias bibliográficas de artículos.

En su análisis de la producción española en Comunicación de 1980 a 2010, de Fernández-Quijada y Masip verifican el impacto del denominado efecto ANECA ya que en el periodo 2004-2008 la producción se dobla (2013: 18). También reseñan que, aunque la mayoría de artículos que se difunden en soportes nacionales están firmados por un solo autor, en 2010 la media de autoría ya se situaba en 1,46 aunque en difusión internacional, en cambio, era de 3,23. En el período analizado, aparecieron 43 revistas de Comunicación en España y desaparecieron 5. En 2013, los datos de DICE registran 52 publicaciones de las que 5 han desaparecido y 6 tienen versión online y en papel, con lo que quedan 41 revistas activas. 


\section{Metodología}

La investigación de los perfiles de investigadores de Comunicación en GSM se desarrolló con una triangulación metodológica y técnicas cuantitativas y cualitativas. Además de la revisión bibliografía para fijar el estado de la cuestión sobre ih en el área de Comunicación se realizó una revisión analítica de los indicadores web de WoK, Scopus y GSM; una exploración de las estadísticas de GSM en las oleadas de 2012 y 2013 y se exploró la creación y el uso de perfiles abiertos en GSM por los investigadores en Comunicación en España. Se solicitó del Ministerio de Educación, Cultura y Deporte un listado de docentes funcionarios de las áreas de Periodismo y de Comunicación Audiovisual y Publicidad para tener un universo (N) referencial de los investigadores del campo de Comunicación. La respuesta del Servicio de Profesorado Funcionario fue denegatoria argumentando que solo podían facilitar "información sobre profesores universitarios cuando ésta es solicitada por el Rectorado de las universidades a los efectos de formación de comisiones juzgadoras". Se optó, entonces, por acudir al listado de miembros de la Asociación Española de Investigación de la Comunicación (AE-IC), la más numerosa de las agrupaciones actuales con 649 socios según el listado publicado en su web en septiembre de 2013. Se rechazaron tres entradas duplicadas y se estableció un Universo de 646 sujetos. Se determinó la selección de una muestra aleatoria con un margen de error muestral del $5 \%$, un nivel de confianza del 97\%, que se fijó en (n) 273 investigadores/as (Tabla 02). Para seleccionar la muestra se uso el listado alfabético de socios en frecuencias de 4 (número de letras del acrónimo de la Asociación). La exploración de perfil se realizó introduciendo en el buscador de GSM el nombre y apellidos del/la investigador/a copiados mecánicamente del listado de a AEIC para evitar errores de transcripción.

\begin{tabular}{|c|c|c|}
\hline FICHA TÉCNICA & Universo N=646 & Muestra (n) $=\mathbf{2 7 3}$ \\
\hline Margen de error $5 \%$ & Nivel de confianza $97 \%$ & Heterogeneidad $50 \% ; \mathrm{p}=\mathrm{q}=50$ \\
\hline Recogida de datos: $4-5 /$ & Análisis de datos: & Base en Excel y exploración con \\
Septiembre/2013 & Septiembre 2013 & SPSS \\
\hline
\end{tabular}

Tabla 02. Ficha técnica de la investigación.

Se parte de hipótesis ciega por no disponer de análisis precedentes. La investigación pretende dar respuesta a: 1) ¿Cuántos investigadores de Comunicación han creado su perfil en GSM?; 2) ¿Cuidan su imagen como investigadores editando sus perfiles?; 3) ¿Cuál es la media de citas de cada investigador?; 4) ¿Que peso real tienen la citas de los últimos cinco años sobre las citas totales?; 5) ¿Cuáles son los indicadores bibliométricos $i h$, ih5 e $i 10$ referenciales de las/los investigadores del área?; y 6)¿cuál es el peso de artículos y libros en las citas?

Los perfiles localizados en GSM se analizaron a través de una ficha de recogida de datos: género, número total de citas, índice h, i10, número de citas en los últimos cinco años, ih5, i10 de los últimos cinco años, edición del guión, fotografía del autor, primer año de referencia en los trabajos incluidos en el perfil y número de trabajos incorporados. Los que aparecen con más de cien publicaciones o con mayor número 
de citas, se someten a una segunda exploración para determinar si los trabajos citado eran artículos o libros.

\section{Resultados}

Uno de cada cinco investigadores $(19,36 \%)$ ha elaborado y tiene en acceso abierto en la red su perfil en GSM. No se disponen de estudios similares en otras áeras para poder contextualizar el dato, aunque si se tiene en consideración el corto espacio de tiempo de vigencia de la aplicación (desde 2012) y el reciente interés del área por el índice h como indicador de la productividad y su impacto, la lectura de un dígito aparentemente bajo pudiera interpretarse en sentido opuesto. La trayectoria media es de 14 años con un h medio de 4 . Los resultados del análisis de perfiles se presentan agrupados por niveles de edición, trayectorias como investigador, citas medias, niveles de producción científica y formato de difusión.

\begin{tabular}{|c|c|c|c|c|c|c|c|c|c|}
\hline \multirow{2}{*}{$\begin{array}{c}\text { Investig. } \\
\text { con perfil }\end{array}$} & \multicolumn{4}{|c|}{ Perfiles editados } & \multirow{2}{*}{ in5 } & $\begin{array}{c}\text { Citas } \\
\text { media }\end{array}$ & $\begin{array}{c}\text { Citas } \\
\text { media } \\
\text { h5 }\end{array}$ & $\begin{array}{c}\text { Primera } \\
\text { publicación } \\
\text { (media) }\end{array}$ \\
\cline { 2 - 8 } & & Total & Datos & Foto & & & & & \\
\hline 19,35 & 14,5 & 15,9 & 84,9 & 33,9 & 4 & 3 & 119,9 & 84,2 & 1998 \\
\hline
\end{tabular}

\subsection{Niveles de edición}

El estudio de los perfiles de socios de la AEIC indica que hay un bajo índice de difusión de la producción científica y académica personal del área de Comunicación en Google Scholar y un aparente desinterés por la edición del propio soporte ya que solo el 14,5\% de los perfiles está editado, es decir, rigurosamente revisados y con los datos personales o de presentación de los trabajos científicos y académicos actualizados (representan un 3\% de la muestra estudiada). Las erratas más frecuentes son: títulos de artículos en mayúsculas o minúsculas; firmas diferentes del mismo autor; entradas tachadas por duplicación de citas que no se han eliminado; atribución de ubicaciones dispares y ausencia de foto. Es representativa esta ausencia de elementos identificativos de relevancia sobre todo tratándose de expertos en Comunicación. Uno de cada tres perfiles $(32,7 \%)$ no tiene rostro (Tabla 03 ).

Los perfiles editados son tan pocos que no permiten lecturas estadísticas pormenorizadas aunque el número de varones con perfil editado cuadriplica al de mujeres en una asociación y una muestra casi equilibrada en peso de ambos géneros. La edición del perfil, que es la imagen pública del investigador, tampoco se asocia a investigadores con mayor productividad. Ninguno de los que sobrepasan la centena de obras en su perfil ha dedicado atención a editar sus datos. 


\subsection{Trayectorias como investigador y media de citas}

Los perfiles ofrecen un panorama transversal de producción e impacto porque se reparten entre investigadores con carreras incipientes y otros con trayectorias consolidadas. Por eso se ha considerado interesante antes de analizar el impacto a través del ih estudiar la distribución y el número de citas globales conseguidas por cada uno. Se han determinado tres grupos de sujetos según la duración de su carrera investigadora, contada a partir del año del primer trabajo científico incorporado a su perfil: De uno a diez años, como investigadores junior, de once a veinte años como investigadores de trayectoria consolidada y con 21 o más años como expertos investigadores sénior. El grueso de la muestra se concentra en la franja media (Tabla 04)

Es lógico que con el tiempo el número de citas aumente por lo que no resulta revelador que la media de citas se incremente en las franjas de edades más altas. Si resulta de interés, no obstante, observar como en los 3 grupos de trayectorias investigadoras se da una situación que se repite: uno de cada tres investigadores tiene un impacto destacado, por encima de la media de su grupo (tabla X). Y, además, es una situación que se pudiera considerar constante a lo largo de toda la trayectoria porque se vuelve a dar al analizar solo las cifras recibidas en los últimos cinco años naturales completos (Tabla 05)

Las citas reflejan los ciclos de productividad. La mayoría de cifras de impacto apreciables se localizan después de diez años como investigador. Incide el ritmo lento de publicación del área y, como se indicó, una reticencia a firmas colectivas de artículos. El cambio de dinámica, seguramente debido al aumento del número de revistas, se observa al comparar el número global de citas con la cantidad de citas de los últimos cinco años. Uno de los resultados más interesantes es comprobar la concentración de impacto del grupo destacado por encima de la media es común a los tres tramos de edad investigadora y progresivo a medida que los años de investigador avanza, sobre todo si se computan aislados los cinco años últimos.

La acumulación de citas se hace notable a partir de los diez años de trayectoria. El cambio de ritmo en los niveles de impacto en los últimos años se hace visible si se comparan las medias de citas de la carrera investigadora con la media de citas logradas solo en ese periodo 2008-2012 (Tabla 06).

\begin{tabular}{|c|c|c|c|c|}
\hline \multirow{2}{*}{$\begin{array}{c}\text { Años desde primer } \\
\text { artículo }\end{array}$} & \multirow{2}{*}{ Investigadores } & \multicolumn{3}{|c|}{ Citas de toda la trayectoria } \\
\cline { 3 - 5 } & & Media & Superan media & Debajo media \\
\hline $1-10$ & 33,3 & 8,5 & 33,33 & 66,66 \\
\hline $11-20$ & 42,6 & 121,6 & 34,78 & 65,22 \\
\hline 21 o más & 24,1 & 262,0 & 38,46 & 61,54 \\
\hline TODAS/OS & 100,0 & 119,92 & \multicolumn{2}{|c|}{} \\
\hline
\end{tabular}

Tabla 04. Citas medias. 


\begin{tabular}{|c|c|c|c|}
\hline \multirow{2}{*}{ Años desde primer artículo } & \multicolumn{3}{|c|}{ Citas últimos cinco años } \\
\cline { 2 - 4 } & Media & Superan media & Debajo media \\
\hline $1-10$ & 8,16 & 33,33 & 66,66 \\
\hline $11-20$ & 92,04 & 39,13 & 60,87 \\
\hline 21 o más & 169,23 & 46,15 & 53,85 \\
\hline TODAS/OS & 84,22 & \multicolumn{2}{|c|}{} \\
\hline
\end{tabular}

Tabla 05. Citas medias últimos cinco años.

\begin{tabular}{|c|c|c|}
\hline Años desde primer artículo & Citas desde 2008 & Citas antes 2008 \\
\hline $1-10$ & 95,5 & 4,5 \\
\hline $11-20$ & 75,7 & 24,3 \\
\hline 21 o más & 64,6 & 35,4 \\
\hline TOTAL & 70,2 & 29,8 \\
\hline
\end{tabular}

Tabla 06. Citas en la trayectoria investigadora.

Hay un pequeño grupo sin impacto, que no se limita a los investigadores más nóveles. Son un pequeño porcentaje $(7,5 \%)$ los que no han logrado ningún reconocimiento de sus pares; entre ellos hay algunos que tienen carreras de solo uno o dos años pero también hay casos de investigadores con ocho y nueve años de trayectoria y sin ningún impacto en los trabajos que han incluido en su perfil.

\subsection{Indices $H$ y $\mathrm{H} 5$}

El índice $h$ medio de los investigadores con perfil es $4(4,30)$, con márgenes extremos en 17 y 0 , y moda igual a 3 . Es significativo que solo un 7,5\% de la muestra tenga $i h \geq 10$ y que el $62 \%$ se sitúe por debajo de un $i h=5$ (Tabla 07 ).

El ih5 es $3(3,7)$ con máximo de 15 , mínimo de 0 y moda de $2-3$, lo que vuelve a incidir en la representatividad de la acción difusora en los últimos años, ya evidenciado al analizar la concentración del número absoluto de citas en el periodo 20082012. Por encima de 10 se sitúa el $2 \%$ y por debajo de 5 , el $72 \%$.

El i10 refuerza, igualmente, esta interpretación ya que el $i 10$ medio de toda la trayectoria investigadora (un $66,7 \%$ de la muestra tiene más de diez años de actividad) es $2(2,56)$ y el $i 10$ medio de los últimos cinco años es 1,64 .

\begin{tabular}{|c|c|c|c|c|c|c|c|}
\hline \multicolumn{4}{|c|}{ Ih } & \multicolumn{4}{|c|}{ ih5 } \\
\hline \multirow{2}{*}{ Media } & \multirow{2}{*}{ Moda } & \multicolumn{2}{|c|}{ i10 } & \multirow{2}{*}{ Media } & \multirow{2}{*}{ Moda } & \multicolumn{2}{|c|}{ i10 } \\
\hline & & Media & Moda & & & Media & Moda \\
\hline $4(4,3)$ & 3 & $2(2,56)$ & 0 & $3(3,69)$ & 2 y 3 & $1(1,64)$ & 0 \\
\hline
\end{tabular}

Tabla 07. Ih e $i 10$ medios. 


\subsection{Nivel de producción}

Solo un 5,5\% de los perfiles encontrados presentan una producción que sobrepasa los cien textos. Una cuarta parte de los investigadores con perfil en GSM tienen toda su producción científica concentrada en el periodo posterior a 2008 (Tabla X). El impacto medio del grupo más productivo es de 3,7 citas por trabajo si se analiza toda la trayectoria y de 2,6 citas por publicación si solo se tienen en cuenta las citas conseguidas en los cinco últimos años (Tabla 08).

\begin{tabular}{|c|c|c|c|c|}
\hline \multirow{2}{*}{ Autores } & \multicolumn{4}{|c|}{ ih5 } \\
\cline { 2 - 5 } & Máximo & Mínimo & Medio & Mediana \\
\hline $28,3 \%$ & 22 & 0 & 9,85 & 7 \\
\hline
\end{tabular}

Tabla 08. Autores con toda la producción desde 2008.

\subsection{El formato: libro \& artículo}

Para aproximarse al peso de los soportes que generan más impacto se analizaron los perfiles de los investigadores con más de cien trabajos publicados y los de aquellos que habían conseguido más de 250 citas al conjunto de sus trabajos y se revisaron sus cuatro producciones con más citas. El resultado solo puede ser global porque GSM no detalla las citas de los últimos cinco años (solo da cifra global e ih5 e i10). La (n) estudiada es muy reducida pero abre una visión interesante: los libros son la mitad pero concentran el doble de citas que las revistas (Tabla 09).

\begin{tabular}{|c|c|c|}
\hline Formato & Citas & Obras \\
\hline Libros & $61,09 \%$ & $37,5 \%$ \\
\hline Revistas & $38,90 \%$ & $62,5 \%$ \\
\hline
\end{tabular}

Tabla 09. Formato de publicación.

Teniendo en cuenta que la mayoría de citas de todos los actores se concentran en los últimos cinco años, se podría interpretar que los libros siguen siendo una referencia de peso en la difusión científica y académica. GSM permite incorporarlos al perfil pero WoK o Scopus solo incorporan artículos de difusión de investigación.

\section{Conclusiones}

De la muestra analizada, uno de cada cuatro investigadores ha abierto su perfil en GSM con su producción científica y académica $(19,36)$, pero más del $80 \%$ han descuidado la edición tanto en aspectos formales de los datos de atribución de los trabajos o de identificación del autor como en aspectos de contenido, con citas tachadas por duplicación y con entradas de un mismo artículo no vinculadas. Hay un deseo de estar y de dar a conocer la actividad de investigación y su repercusión pero no se cuida la imagen rigurosa del investigador a través de elaboración del perfil, lo que es 
significativo en un área como Comunicación. Se responde por tanto a las preguntas de investigación 1 y 2 .

El ih referencial del área según la fórmula de Imperial y Navarro es 17; el índice máximo de las revistas del área en GSM es 13. En los perfiles de la muestra analizada. Los ih que igualan o superan 10 solo son el 7,5\%. La media es significativamente reducida, 4, (ih5 medio es 3) que es el índice de las revistas de Comunicación de tercer y cuarto quartil en el listado internacional de EC3, 2013. Se responde la pregunta 5 .

La actividad de investigación se activa en los últimos años. La difusión e impacto que se registra a través de los perfiles está concentrada en el último quinquenio, como evidencia la comparativa del número de citas recibidas en toda la carrera y los de los últimos cinco años, con una concentración del 70,2\% en las referencias desde 2008. Se responden las preguntas 3 y 4 .

A pesar del auge de difusión y del incremento del número de revistas del área, las obras que acumulan mayores impactos siguen siendo los libros. El análisis de los principales trabajos de los investigadores con mayor número de citas o con mayor ih revela que su repercusión se centra un 39\% en libros mientras las citas que provienen de artículos en revistas son el $61 \%$. Se responde así la pregunta de investigación número 6 .

El uso de ih e ih5 como referencias bibliométricas en perfiles de GSM individualiza las métricas y las difunde en abierto. El perfil activo es la primera entrada en la lista de enlaces que ofrece Google Académico cuando se teclea en su buscador el nombre del autor, lo que sugiere la necesidad de derivar a tarea compartida entre revista y autor la visibilidad de la investigación y a incorporar la gestión integral de comunicación de investigación como una parte de los proyectos.

\section{Bibliografía}

DELGADO LÓPEZ-COZAR, Emilio, MARCOS CARTAGENA, Diego, JIMÉNEZ-CONTRERAS, Evaristo y RUIZ-PÉREZ, Rafael, "Índice H de las revistas españolas de Ciencias Sociales y Jurídicas según Google Scholar (2002-2011)", EC3 Informes, $n^{\circ}$ 4, 29 de mayo de 2013, pp. 36.

DELGADO, Emilio; REPISO, Rafael. "El impacto de las revistas de comunicación: comparando Google Scholar Metrics, Web of Science y Scopus". Comunicar nº1, v. XXI, pp. 45-52. DOI: http://dx.doi.org/10.3916/C41-2013-04 ]

FERNÁNDEZ-QUIJADA, David; MASIP, Pere (2013). "Tres décadas de investigación española en comunicación: hacia la mayoría de edad", Comunicar $\mathrm{n}^{\circ} 41$, v. XXI, pp 15-24. DOI: http://dx.doi.org/10.3916/C41-2013-01

HIRSCH, Jorge E., "An index to quantify an individual's scientific output", Proceedings of the National Academy of Sciences of the United States of America, vol. 102, 2005, pp. 16569-16572. 
IMPERIAL, Juan y RODRÍGUEZ-NAVARRO, Alonso, "Usefulness of hirsch's h-index to evaluate scientific research in Spain", Scientometrics, vol. 71, $\mathrm{n}^{\mathrm{o}} 2$, 2007, pp. 271-282.

REPISO, Rafael y DELGADO LÓPEZ-COZAS (2013). "H Index Communication Journals according to Google Scholar Metrics (2008-2012)". EC3 Reports, 6 : 26 octubre 2013. http://ec3noticias.blogspot.com.es/2013/10/publicado-el-indice-h-de-las-revistas.html [31-10-2013]

SÁNCHEZ PITA, F. (2012): "Actualización del Î́ndice H de las revistas de Comunicación, según Google Scholar Metrics", Actas. IV Congreso Int. Latina de Comunicación Social. La Laguna, http://www.revistalatinacs.org/12SLCS/2012 actas/209_Pita.pdf [04-09-2013]

TÚÑEZ LÓP̄EZ, Miguel, "Los periódicos en las redes sociales: audiencias, contenido, interactividad y estrategias comerciales", Estudios del Mensaje Periodístico, vol. 18, 2012, p. 224.

TÚÑEZ, Miguel y DE PABLOS, José Manuel, El 'índice h' en las estrategias de visibilidad, posicionamiento y medición de impacto de artículos y revistas de investigación, Actas del II Congreso Nacional sobre Metodología de la Investigación en Comunicación, Universidad de Valladolid, mayo 2013, pp. 133-150. http:// uvadoc.uva.es/handle/10324/2996.

TÚÑ̃Z, Miguel, "El 'índice h' de la investigación en Comunicación en España, Portugal y Latinoamérica: Web of Knowledge (WoK), Scopus y Google Scholar Metrics". Communication and Society/Comunicación y Sociedad, vol. XXVI, n. 4, 2013, pp.53-75.

\section{El autor}

José Miguel Túñez López. Doctor en periodismo por la Universidad Autónoma de Barcelona. Profesor de Comunicación Organizacional en la Facultad de Ciencias de la Comunicación de la Universidad de Santiago de Compostela. Miembro del Grupo de Investigación Novos Medios. Entre sus publicaciones más recientes: La Gestión de la Comunicación en las Organizaciones, Comunicación Social Ediciones: Zamora/ Sevilla, 2013. 